\title{
Gender differences in the clinical features of hypertrophic cardiomyopathy caused by cardiac myosin-binding protein $\mathrm{C}$ gene mutations
}

Yasunobu Terauchi (MD), Toru Kubo (MD,PhD, FJCC), Yuichi Baba (MD, PhD), Takayoshi Hirota (MD, PhD), Katsutoshi Tanioka (MD), Naohito Yamasaki (MD, PhD), Takashi Furuno (MD, PhD), Hiroaki Kitaoka (MD, PhD, FJCC)

From the Department of Cardiology, Neurology and Aging Science, Kochi Medical School, Kochi University, Kochi, Japan

Hypertrophic cardiomyopathy $(\mathrm{HCM})$ is a primary and genetically transmitted myocardial disorder characterized by thickening of the left ventricular (LV) wall in the absence of another cardiac or systemic disease capable of producing the magnitude of evident hypertrophy. HCM is usually caused by sarcomere protein gene mutations, and the cardiac phenotype has great diversity in morphologic features, age of onset, and clinical course. This phenotypic heterogeneity suggests the existence of some factors that modify the disease presentation. Although gender may be one of the important factors modifying phenotypic expression in HCM, there has been little information on it. The purpose of this study was to clarify the gender differences in clinical features of 
familial HCM caused by cardiac myosin-binding protein C gene (MYBPC3) mutations.

\section{Methods and Results}

We investigated gender differences in the clinical features of $\mathrm{HCM}$ caused by MYBPC3 mutations. Sixty-one subjects (28 families) carrying MYBPC3 mutations were studied. Of 61 subjects with MYBPC3 mutations, fifty patients including 23 female patients were phenotype-positive by echocardiography. Disease penetrance in subjects aged $\leq 40$ years old was $92 \%$ in males and $67 \%$ in females. Females showed delayed onset of left ventricular hypertrophy compared with males in subjects who were genotype-positive. Female patients were more symptomatic at diagnosis than were males (mean New York Heart Association class: $1.7 \pm 0.8$ versus $1.2 \pm 0.4, p=0.012$ ). From a longitudinal point of view by age, no significant gender difference in cardiovascular deaths or cardiovascular events was found. During the follow-up period after diagnosis of HCM (13 \pm 8 years), female patients who were phenotype-positive had significantly more frequent heart failure events than did phenotypically affected male patients $(p=0.028)$.

\section{Discussion:}

This study confirmed a high cardiac disease penetrance in MYBPC3 mutation carriers in females and males. Furthermore, the age-dependent penetrance of LVH was even 
higher in men than in women. Women may have some mechanisms, such as genetic (modifier genes on the sex chromosome) and sexual hormonal factors, that prevent the development of hypertrophy, resulting in later onset of disease.

Compared to male patients, female patients were more symptomatic at diagnosis in our genotyped cohort. Furthermore, from a longitudinal point of view by age, no significant gender difference in HCM-related adverse events was found in either genotype-positive patients or phenotype-positive patients. However, during the follow-up period from after diagnosis of HCM, once LV hypertrophy had developed, female patients had significantly more frequent heart failure events than did phenotypically affected male patients. The reason for these findings is not obvious.

There are several limitations to be acknowledged in the present study. First, due to the retrospective design of the study, it is possible that there is a selection bias. Second, multivariate analysis was not used to evaluate the prognostic values of these clinical profiles because of the small sample numbers. Third, because we focused on HCM patients with MYBPC3 mutations, it is not known whether the same results would be obtained for patients with mutations in other sarcomere genes. To clarify the gender differences as the important modifying factors in HCM, large scale studies examining the relation between genotype, penetrance, disease severity, and prognosis are 
needed.

\section{Conclusions}

Although females with MYBPC3 mutations showed later onset of the disease, female patients were more symptomatic at diagnosis and had more frequent heart failure events once they had developed hypertrophy. 